\title{
Compassion Fatigue among Indian Physiotherapists: a descriptive cross sectional comparative study
}

\author{
Poovishnu Devi T. ${ }^{1}$, Chitra Khanwelkar ${ }^{1}$, Ajay Patil ${ }^{1}$, Supriya S. Patil ${ }^{2}$, Renuka Pawar ${ }^{3}$, \\ Deepika Suruve ${ }^{4}$, S.K. Mohana Sundari ${ }^{5}$ \\ ${ }^{1}$ Bioethics and Research Unit of the UNESCO Chair in Bioethics at Krishna Institute of Medical Sciences \\ Deemed to be University, Karad, Maharashtra. \\ ${ }^{2}$ Associate Professor, Department Of Community Medicine, KIMSDU, Karad. \\ ${ }^{3}$ Professor, Department Of Orthodontics, School Of Dental Sciences, KIMSDU, Karad. \\ ${ }^{4}$ Intern, Faculty of Physiotherapy, KIMSDU, Karad. \\ ${ }^{5}$ Tutor, College of Nursing, AIIMS, Jodhpur. \\ Corresponding Author: Poovishnu Devi T. \\ E-mail: vishnudevi25@yahoo.co.in
}

\begin{abstract}
Background: Compassion has been variously defined as a feeling, attitude or trait arising in witnessing others distress and that motivates a subsequent desire to help. Compassion fatigue is used to describe potential emotional impact on professionals working in trauma care, rehabilitation of degenerative disease and end of life care diseases. The role of physiotherapists is very crucial as they rehabilitate irreversible conditions, manage maintenance phase in degenerative diseases and prevent progression of the disease. This study focuses on assessing compassion fatigue among Indian physiotherapists working in different setups.

Methods: A descriptive cross-sectional comparative survey was conducted among Indian physiotherapists working in ICU setup, rehabilitation setup and general OPD setup by purposive sampling. A total of 520 physiotherapists participated in the survey conducted using survey monkey. ProQOL-5 scale is used to measure the outcomes. The data was analyzed by SPSS software version 16 .

Results: When compared with the work set up ,physiotherapists working in rehabilitation had more compassion fatigue $(p=0.035)$ than the ICU setup followed by OPD services, Male gender had more compassion fatigue $(\mathrm{p}=0.130)$ and physiotherapists completed postgraduation along with special training had better compassion $(p=0.023)$ while physiotherapists clinical experience ranging from $0-10$ years had more compassion fatigue $(p=0.003)$ compared with above 10 years experienced .
\end{abstract}

Conclusion: Compassion fatigue is an important construct that must be evaluated and incorporated in the training of physiotherapists.

Key words: Compassion Fatigue, Compassion satisfaction, Burn out, Indian physiotherapists, secondary traumatic stress.

\section{INTRODUCTION}

Physiotherapy care focuses primarily from ICU to tertiary care in rehabilitation both hospital and community based which proceeds months to years unruffled and requires kindness, compassion and competency. Physiotherapists remain perennial responders to the traumatized patients with high chance to internalize their stress and get pretentious by compassion fatigue [1]. Compassion fatigue express symptoms like hopelessness, lack of pleasure, anxiety, stress, sleeplessness and a 
negative attitude towards life. This decreases self-efficacy and confidence leading to deterioration in performance and work output [2].

Multiple factors are involved in the pathophysiology of compassion fatigue. Personal factors are level of sympathy and compassion, age, gender, ideology and personality type. External or environmental factors are job related stress, support from society, family and friends, ethnicity and training. Having any one or both predisposes a high risk of developing compassion fatigue [3].

Compassion fatigue is comprised of two parts: burnout, characterized as "exhaustion, frustration, anger and depression" [4], and secondary traumatic stress, described as the negative consequences secondary to fear and work-related trauma. Compassion satisfaction is the positive feelings derived from helping others, whether it can be from direct contribution or for the betterment of society [5]. Although compassion fatigue is often used synonymously with burnout, the two concepts are derived from two separate failed survival strategies. Compassion fatigue arises from a rescuecaretaking response, and burnout arises from an assertiveness-goal achievement response [6]. Compassion fatigue occurs when the caretaker cannot shield or save the individual from harm and, therefore, results in feelings of guilt and distress, and burnout is when one cannot achieve an anticipated goal, resulting in frustration and perceived loss of control [7]. Compassion fatigue is caused by a natural and intrinsic response to alleviate pain and suffering. Burnout is environmentally driven (e.g., time and resource constraints, increased workload) [8] and the onset and resolution period between compassion fatigue and burnout are different. Compassion fatigue has an acute and insidious onset, resulting in long-term consequences that are not easily reversible. Conversely, burnout has a rapid onset and resolution, suggesting that removal of stressor source may be effective [9]. A study conducted in nurses showed that they felt as they have become pessimistic, anergic, and less sympathetic towards their patients and even realized as they were distancing from patients and even from their colleagues [10]. Many studies showed that specialty, duration spent with patients and treatment outcomes has a profound impact on developing compassion fatigue [11]. Till date research on compassion fatigue is not yet been conducted among Indian physiotherapists who are tortuous in treating long term conditions (stroke, spinal cord injuries, cerebral palsy), progressive conditions (COPD, cancer, heart failure) and degenerative conditions (muscular dystrophy, Parkinson's disease, arthritis) and remain associated with the patient for hours and also have an elusive bonding with patients.

To confront the problem of compassion fatigue, first it should be properly assessed based on their profession not in a wide view of health care profession, sound planning parameters need to be developed for which basic data from each own set up in needed, the current study is planned for to assess the compassion fatigue, among Indian physiotherapists working in different setups.

The aim of the current study was to assess compassion fatigue among Indian physiotherapists working in different setups, to determine compassion fatigue among Indian physiotherapists working in ICU setup, to determine compassion fatigue among Indian physiotherapists working in rehabilitation and OPD setup and to identify which demographic and work related variable contribute to the risk for compassion fatigue among Indian physiotherapists

\section{METHODOLOGY}

A survey conducted among Indian physiotherapists working in ICU, rehabilitation and general OPD setup. Duration of the study was six months. A total of 580 participants were selected in the study through non-probability purposive sampling. Finally 35 respondents could not complete the questionnaire leaving a sample size of 545. The participants included a) physiotherapists working in ICU, OPD and rehabilitation services, b) Both male and female physiotherapists c) physiotherapist holding minimum bachelor's degree. Physiotherapists, not directly related to patient care, were excluded from the study.

\section{Procedure}

After getting approval from institutional protocol and ethical committee the survey was sent to the selected physiotherapists covering most parts of India through mail. The survey monkey, a webbased survey tool for data collection, was used to conduct the survey. It is designed with inclusion 
of demographic data and professional questions. Clicking the continue option from demographic data to the survey is taken as informed consent for the participants. Once the survey was forwarded after 2 weeks a remainder mail is sent and a subsequent remainder after one week is sent.

\section{Data analysis}

The collected data was compiled for analysis. The obtained sample was coded and analyzed in SPSS version 16.0. Descriptive statistics was used for calculating mean and standard deviation for continuous variables whereas frequency and percentage for categorical variables. Inferential statistics was used for association and comparison among different variables. Reliability of the test was assessed through internal consistency by applying Cronbach's alpha test. Item analysis was performed by mean \pm standard deviation of each item along with reliability analysis. The $\alpha$ was set as less than 5 for significance at a confidential level of $98 \%$

\section{RESULTS}

545 physiotherapists participated in the study at a response rate of about $93 \%$. Reliability of the question was determined by Cronbach's alpha test which was calculated to be 0.81 showing higher reliability.

Table 1: Frequency, percentage and comparison of different levels of compassion fatigue in three different setups

\begin{tabular}{|c|c|c|c|c|}
\hline \multirow{2}{*}{$\begin{array}{c}\text { Compassion } \\
\text { fatigue level }\end{array}$} & \multicolumn{3}{|c|}{ Work setup (n=545) } & \multirow{2}{*}{ p value } \\
\cline { 2 - 4 } & $\begin{array}{c}\text { ICU } \\
(90)\end{array}$ & $\begin{array}{c}\text { Rehabilitation } \\
(190)\end{array}$ & $\begin{array}{c}\text { OPD } \\
(165)\end{array}$ & \\
\hline Low & $30(33.33 \%)$ & $56(29.47 \%)$ & $71(43.03 \%)$ & \multirow{2}{*}{$0.035^{*}$} \\
\hline Average & $53(58.8 \%)$ & $120(63.15 \%)$ & $90(54.54 \%)$ & \\
\hline High & $7(7.77 \%)$ & $14(7.36 \%)$ & $4(2.42 \%)$ & \\
\hline
\end{tabular}

*significant $(\mathrm{P}<0.05)$

Compassion fatigue was compared between physiotherapists working in rehabilitation setup, ICU setup and general OPD setup. Chi square test showed significant difference in compassion fatigue among the three categories ( $\mathrm{p}=0.035)$ with high compassion fatigue among the rehabilitation setup physiotherapists followed by ICU therapists.

Table 2: Frequency and percentage and comparison of different levels of compassion fatigue between males and females

\begin{tabular}{|c|c|c|c|}
\hline $\begin{array}{c}\text { Compassion fatigue } \\
\text { level }\end{array}$ & Male (390) & Female (155) & p value \\
\hline Low & $160(4 \%)$ & $63(40.64 \%)$ & \multirow{2}{*}{0.130} \\
\hline Average & $213(46.15 \%)$ & $82(52.90 \%)$ & \\
\hline High & $17(4.35 \%)$ & $10(6.45 \%)$ & \\
\hline
\end{tabular}

Compassion fatigue was compared between the two categories of gender, male and female. Chi square test showed difference in compassion fatigue between the two categories $(p=0.130)$ but not statistically significant with high compassion fatigue among the males compared to females. 
Table 3: Frequency and percentage and comparison of different levels of compassion fatigue based on experience

\begin{tabular}{|c|c|c|c|c|c|}
\hline \multirow{2}{*}{$\begin{array}{c}\text { Compassion } \\
\text { fatigue level }\end{array}$} & \multicolumn{3}{|c|}{ Experience $(\mathrm{N}=545)$} & \multirow{2}{*}{$\mathrm{p}$ value } \\
\cline { 2 - 5 } & $\begin{array}{c}0-5 \text { years } \\
(130)\end{array}$ & $\begin{array}{c}5-10 \text { years } \\
(255)\end{array}$ & $\begin{array}{c}10-15 \text { years } \\
(100)\end{array}$ & $\begin{array}{c}15 \text { years and } \\
\text { above } \\
(60)\end{array}$ & \\
\hline Low & $29(22.3 \%)$ & $55(23.52 \%)$ & $35(35 \%)$ & $30(50 \%)$ & \multirow{2}{*}{$0.023^{*}$} \\
\hline Average & $78(60 \%)$ & $154(58.43 \%)$ & $55(55 \%)$ & $22(36.6 \%)$ & \\
\hline High & $23(17.69 \%)$ & $46(18.03 \%)$ & $10(10 \%)$ & $8(13.33 \%)$ & \\
\hline
\end{tabular}

*significant $(\mathrm{p}<0.05)$

Compassion fatigue was compared between four categories of physiotherapists' experience i.e. from 0-5 years, 5-10 years, 10-15 years and 15 years and above. Chi square test showed significant difference in compassion fatigue among the four categories $(\mathrm{p}<0.023)$ with high compassion fatigue among the 0-5 years experienced physiotherapists followed by 5-10 years physiotherapists.

Table 4: Frequency and percentage and comparison of different levels of compassion fatigue based on educational qualification

\begin{tabular}{|c|c|c|c|c|c|}
\hline \multirow[t]{2}{*}{$\begin{array}{l}\text { Compassion } \\
\text { fatigue level }\end{array}$} & \multicolumn{4}{|c|}{ Educational Qualification } & \multirow[t]{2}{*}{$\mathrm{p}$ value } \\
\hline & $\begin{array}{l}\text { BPT } \\
(210)\end{array}$ & $\begin{array}{l}\text { MPT } \\
(255)\end{array}$ & $\begin{array}{c}\text { MPT with } \\
\text { special training } \\
\text { (72) }\end{array}$ & $\begin{array}{c}\text { Ph D } \\
(8)\end{array}$ & \\
\hline Low & $5(2.38 \%)$ & $57(22.35 \%)$ & $41(54.66 \%)$ & $1(12.5 \%)$ & \multirow{3}{*}{$0.033^{*}$} \\
\hline Average & $85(40.47 \%)$ & $112(43.92 \%)$ & $12(16.66 \%)$ & $3(37.5 \%)$ & \\
\hline High & $120(57.14 \%)$ & $86(33.72 \%)$ & $19(26.38 \%)$ & $4(50 \%)$ & \\
\hline
\end{tabular}

*significant

Compassion fatigue was compared between four categories of physiotherapist qualification. Chi square test showed significant difference in compassion fatigue among the four categories $(\mathrm{p}<$ 0.033) with high compassion fatigue is among physiotherapists holding Bachelor's degree alone followed by $\mathrm{PhD}$ holders and Master's degree

\section{DISCUSSION}

Stamm's guidelines was used to calculate patients score and categorise into low, average and high levels of compassion fatigue. From the above results it is shown that compassion fatigue is more in physiotherapists working in rehabilitation setup .Patients in need of palliative care, rehabilitation after cardiac and pulmonary surgeries and heart failure patients need more thoughtfulness and deliberation as each of the patient is unique specifically related to suffering and each should be addressed in compassion that can offer substantial benefit for patients such as patient therapist relation patient satisfaction, improvement in symptoms and promote quality of life and aid recovery or else it will result in progressive deterioration of health .So the huge affliction burdens the physiotherapists and treating same kind of patients daily may also result in compassion fatigue

Researchers studied 681 health care professional providers and reported that high CF scores were associated with the nature of work place and the number of years worked as health care professional and concluded that the CF scores became worsened over time. The word 'satisfied' 
from 'fatigued' participants were the ability to deal with high work load and having positive view about the team with which they worked. The above study results are in keeping with the results of the present study [13].

Compassion fatigue is more in males compared to in females despite the type of work set up because of cultural effect as they may not sensitively be involved with the patients and male population has experienced ten times more compassion fatigue than female population and practicing females were less compared to male in India but the results are reverse in western population [14].

Compassion fatigue is more common in less experienced therapists that is from $0-10$ years as during the initial and developing years the therapists must be finding it difficult in managing time, more work burden, selection of better techniques and lack of practice also thrust them into compassion fatigue compared to senior physiotherapists as they might have mastered in coping the problems related to patients

Compassion fatigue is experienced more in undergraduate physiotherapists as they lack specialisation and special training. Physiotherapists with post-graduation and special training with experience showed a less compassion fatigue compared with post graduate only and doctorate physiotherapists.

Authors studied with 151 freshly passed out physiotherapists and the study exhibited high level of compassion satisfaction, by understanding the experience of physiotherapists, we can better anticipate the support needed for the newer graduates to overcome the struggle which results in compassion fatigue [15].

\section{Limitations}

- Only one outcome tool is used in the study

- Small sample size

- Cross sectional study

- Purposive sampling reduces generalizability

\section{CONCLUSION}

Compassion fatigue affects both physical and mental wellness and results in reduced quality work output. Physiotherapists in rehabilitation services and critical care should be deloaded at regular intervals and necessary training should be given to deliver the service to patient satisfaction.

\section{REFERENCES}

1. Bellolio MF, Cabrera D, Sadosty AT, Hess EP, Campbell RL, Lohse CM. Compassion Fatigue is Similar in Emergency Medicine Residents Compared to other Medical and Surgical Specialties. West J Emerg Med 2014;15(6):629-35.

2. Sung K, Seo Y, Kim JH. Relationships between compassion fatigue, burnout, and turnover intention in Korean hospital nurses. J Korean Acad Nurs 2012;42(7):1087-94.

3. Lombardo B, Eyre C. Compassion fatigue: a nurse's primer. Online J Iss Nurs 2011;16(1):3.

4. Schantz M. Compassion: a concept analysis. Nurs Forum 2007;42:48-55.

5. van der Cingel M. Compassion: the missing link in quality of care. Nurse Educ Today 2014;34:1253-7.

6. Sinclair S, Norris JM, McConnell SJ, et al. Compassion: a scoping review of the healthcare literature. J Pain Symptom Manage BMC Palliat Care 2016;15:6-20.

7. Sinclair S, McClement S, Raffin Bouchal S. Compassion in healthcare: an empirical model. J Pain Symptom Manage 2016;51(2):193-203.

8. Frampton SB. Compassion as the foundation of patient-centered care: the importance of compassion in action. J Comp Eff Res 2013;5(3):443-55.

9. Heyland DK, Dodek P, Rocker G. Canadian Researchers End-of-Life Network (CARENET). What matters most in end-of-life care: perceptions of seriously ill patients and their family members. CMAJ 2006;174(5):627-33. 
10. Riggs JS, Woodby LL, Burgio KL. "Don't get weak in your compassion": bereaved next of kin's suggestions for improving end-of-life care in Veterans Affairs Medical Centers. J Am Geriatr Soc 2014;62(4):642-4

11. Back AL, Deignan PF, Potter PA. Compassion, compassion fatigue, and burnout: key insights for oncology professionals. American Society of Clinical Oncology educational book / ASCO American Society of Clinical Oncology Meeting. 2014: e454-9.

12. Figley CR. Treating compassion fatigue. Routledge: UK; 2002.

13. Lauvrud C, Nonstad K, Palmstierna T. Occurrence of post traumatic stress symptoms and their relationship to professional quality of life (ProQoL) in nursing staff at a forensic psychiatric security unit: a cross-sectional study. Health Qual Life Outcomes 2009;7:31-7.

14. Dasan S, Gohil P, Cornelius V, Taylor C. Prevalence, causes and consequences of compassion satisfaction and compassion fatigue in emergency care: a mixed-methods study of UK NHS Consultants. Emerg Med J 2014;18.

15. Slocum-Gori S, Hemsworth D, Chan WW, Carson A, Kazanjian A. Understanding Compassion Satisfaction, Compassion Fatigue and Burnout: a survey of the hospice palliative care workforce. Palliat Med 2013;27(2):172-8.

16. Klappa SG, Howayek KR. Compassion fatigue among new graduate physical therapists. Global J Med Phys Health Educ 2015;2:100-11

Acknowledgements - Nil

Source of Funding - Nil

Conflict of Interest - Nil 